\title{
Gleiche Würm-Rückzugsstadien in den Gebirgen Mitteleuropas und Ostafrikas?
}

\author{
Von Adolf Zienert, Heidelberg \\ Mit 1 Abbildung und 2 Tabellen
}

$\mathrm{Zus}$ a mme n fassung. Vergleiche der von B. H. BAKer gefundenen Gletscherstände am Mit. Kenya mit der Karte 1:25000 des Mt. Kenya und mit den Würm-Rückzugsstadien der alpinen Gletscher ergeben interessante Anregungen für die weitere Erforschung dieses Fragenkomplexes.

$\mathrm{S} \mathrm{u} \mathrm{m} \mathrm{m}$ a r y . B. H. BAKERs paper of 1967 concerning the geology of the Mount Kenya area containes detailed data about the moraines. Nine stages beginning with würm-terminal could be destinguished beneath the small glaciers of today. By means of an exact examination of the facts the map $1: 25000$ shows the number of moraines known with great probability could be almost doubled by the author of this paper. All these moraines fit into BAKERs system. At the Kibo (Kilimanjaro) the system seems to be less complete (Ch. Downie 1964). On the other hand the extension of the depression of the "snow-line" of each one of these stadials fits in its tendency very well into the system of the postwürm stadials of the Alps. But: for exact correlation regarding the stadials of the mountains in the east-african tropics with those of the Alps it will be necessary to examine the same problem otherwhere in the world.

Bisher scheint über die Rückzugsstadien der letzten Vereisung in tropischen Gebirgen nicht allzuviel bekannt gewesen zu sein, da man sich hauptsächlich mit der Frage nach dem Maximalstand der Gletscher beschäftigte. Als um so wertvoller und anregender dürfte sich deshalb die neue Untersuchung von BAKER über die Moränen des M. Kenya erweisen, die im Rahmen seiner "Geology of the Mount Kenya Area" 1967 veröffentlicht wurde.

B. H. BAKER konnte, unterhalb der heute recht kleinen Gletscher des M. Kenya, Moränen von insgesamt ne un $\mathrm{S}$ tadien unterscheiden (bei ihm und im Folgenden: IAID und II-VI), die manchmal noch aus mehreren Wällen bestehen. Eine genaue Durcharbeitung der Untersuchung von BAKER an Hand seiner Kartenbeilagen und besonders der schönen topogr. Karte 1:25000 des Mount Kenya (nur der Bereich der Stände IA-D liegt meist außerhalb dieses Kartenblattes) ergab, daß sich allein durch die Auswertung dieses Blattes die Zahl der von BAKER gefundenen Gletscherstände nicht nur voll bestätigen läßt, sondern auch noch in von ihm wohl nicht näher untersuchten Tälern auf Grund alpiner und Mittelgebirgserfahrung in der Auswertung von Karten eine ganze Reihe weiterer Moränen mit größter Wahrscheinlichkeit auffinden läßt, die alle in die von B. H. BAKER entwickelte Gliederung der Rückzugsstadien hineinpassen (Tab. 1 und Diagramm 1; sie enthalten die Gletscherstände der 20 wichtigsten Täler des M. Kenya von NW an im Uhrzeigersinn aufgezählt).

Schaut man sich das Diagramm 1 näher an, so ergibt sich zunächst einmal, welche Moränen BAKER fand ( + ), und welche zusätzlich von mir auf der Karte 1:25000 aufgefunden werden konnten ( - und $\sim$ ), außerdem der Gletscherstand von etwa 1960. Man kann daraus aber noch mehr ablesen. Die Gletscher waren dort am längsten, wo das Einzugsgebiet bis zu den Hauptgipfeln reichte, je niedriger der höchste Punkt des Einzugsgebietes, desto kürzer der Gletscher darunter. Gelegentlich kommen Ausnahmen von dieser Regel vor, und zwar da, wo die Gletscher über sonst trennende $Z$ wischentalrücken überfließen konnten (vor allem in IA-III beiderseits vom Teleki-Tal und im Bereich des Ruguti-Tales). Eigentliche Expositionsunterschiede treten nicht auf, sind auch bei der Lage wenige $\mathrm{km}$ südlich des Äquators nicht zu erwarten. Es lassen sich aus dem Diagramm Durchschnittswerte für die Höhenlage der Moränen ablesen (Ausnahmen: IB und 
Tabelle 1: Kenya-Moränen

\begin{tabular}{|c|c|c|c|c|c|c|c|}
\hline $\begin{array}{l}\text { Tal- } \\
\text { No. }\end{array}$ & IA & IB & IC & ID & II & III & IV \\
\hline 1 & $9.200-9.700 ?$ & $?$ & $?$ & $?$ & 11.800 & ? & $\begin{array}{c}13.370-13.700 \\
(3 \text { Wälle })\end{array}$ \\
\hline 2 & $?$ & $?$ & $?$ & $?$ & $?$ & $\begin{array}{l}12.900-13.000 \\
(2 \text { Wälle })\end{array}$ & 13.400 \\
\hline 3 & $10.600-10.800 ?$ & $?$ & $?$ & $?$ & 12.450 & 13.000 & $?$ \\
\hline 4 & ? & ? & $?$ & $12.300\left(?={ }_{n} \mathrm{~A}^{\prime \prime}\right)$ & $?$ & 13.100 & $\begin{array}{l}\text { ? } 13.500 \\
\quad \text { (nur Boden) }\end{array}$ \\
\hline 5 & 11.900 & 12.050 & 12.120 & 12.300 & 12.650 & 13.200 & $\begin{array}{l}\text { ? } 13.700 \\
\quad \text { (zwei Böden) }\end{array}$ \\
\hline 6 & $11.200-11.300 ?$ & - & - & $11.600(=\mathrm{B}-\mathrm{D} !)$ & 12.500 & $13.000-13.200$ & 13.750 \\
\hline 7 & 11.100 & 11.300 & 11.700 & 11.900 & 12.500 & $13.050 ?$ & $?$ \\
\hline 8 & $10.200-10.300 ?$ & 10.600 & 10.900 & $\begin{array}{l}11.400 \text { (mehrere } \\
\text { Wälle) }\end{array}$ & $12.650 ?$ & $\begin{array}{l}\text { ? (in der } \\
\quad \text { Schlucht ?) }\end{array}$ & 14.200 \\
\hline 9 & ? & $?$ & $?$ & $11.800\left(?={ }_{\#} \mathrm{~A}^{\prime \prime}\right)$ & 12.600 & 13.600 & - \\
\hline 10 & 10.000 (n. Karte) & 10.400 & $?$ & $11.200-11.300$ & 12.200 & $12.800-13.100$ & $14.200-14.250$ \\
\hline 11 & $10.500-10.600 ?$ & $?$ & 11.300 & 11.900 & 12.350 & $?$ & - \\
\hline 12 & $10.300-10.400 ?$ & $?$ & 10.800 & 11.100 & 11.800 & $\begin{array}{l}\text { 12.750-13.000 } \\
\quad \text { (Carr's Lakes) }\end{array}$ & $\begin{array}{l}14.000 \text { (Thompon } \\
\text { Tarn) }\end{array}$ \\
\hline 13 & $11.000 ?$ & $?$ & $?$ & 11.900 & 12.800 & $\begin{array}{l}13.400 \\
\quad(2 \text { Wälle) }\end{array}$ & 13.950 \\
\hline 14 & ? & $?$ & $?$ & $?$ & 12.900 & 13.500 & $\begin{array}{l}13.950 \text { (Hidden } \\
\text { Tarn) }\end{array}$ \\
\hline 15 & $?$ & ? & $?$ & $?$ & 12.900 & 13.500 & 13.800 \\
\hline 16 & $11.450 ?$ & $11.600 ?$ & $?$ & 12.000 & 12.900 & $?$ & - \\
\hline 17 & $9.700 ?$ & $?$ & $10.400 ?$ & $10.700 ?$ & $11.600 ?$ & $12.600 ?$ & $\begin{array}{c}13.750 \text { (Lake } \\
\text { Höhnel) }\end{array}$ \\
\hline 18 & $10.000-10.200 ?$ & 10.400 & $10.850 ?$ & 11.200 & 12.400 & 12.900 & $\begin{array}{c}\text { 13.570-13.750 } \\
\text { (6 Wälle) }\end{array}$ \\
\hline 19 & $9.750 ?$ & $?$ & $?$ & $11.000 ?$ & $\begin{array}{r}12.100- \\
12.250\end{array}$ & 12.750 & $\begin{array}{c}13.300-13.550 \\
(3 \text { Wälle })\end{array}$ \\
\hline 20 & $10.500 ?$ & $?$ & $?$ & 11.250 & $?$ & $12.750-12.850$ & $?$ \\
\hline \multicolumn{8}{|c|}{ Durchschnittswerte nach Diagramm 1: } \\
\hline- & 10.500 & & & 11.600 & 12.300 & 13.150 & 13.800 \\
\hline \multicolumn{2}{|c|}{$\Delta$ in Fuß: } & 1.100 & & 700 & 850 & 650 & 600 \\
\hline \multicolumn{2}{|c|}{$\Delta / 2$ in Meter: } & $165 \mathrm{~m}$ & & $105 \mathrm{~m}$ & 130 & $100 \mathrm{~m}$ & $90 \mathrm{~m}$ \\
\hline
\end{tabular}

A n merkungen:

Die entsprechenden Moränen kann auf Grund der Höhenangabe jeder glaziologisch Geschulte unschwer in der Karte 1:25000 des Mount Kenya auffinden; soweit sie in wenigen Ausnahmen dort nicht ohne Weiteres erkennbar sind, handelt es sich um einwandfrei von BAKER kartierte Moränen, z. B. IV und V im Teleki-Tal. 
(Abb. 1; Höhen in Fuß)

\begin{tabular}{|c|c|c|c|c|}
\hline $\mathrm{V}$ & VI & $\begin{array}{l}\text { Gletscher-Stand } \\
1960=\text { "VII“ }\end{array}$ & Gipfelhöhe & $\begin{array}{l}\text { Fluß oder Tal } \\
\text { (V. = Valley) }\end{array}$ \\
\hline 13.900 & $\begin{array}{l}14.400-14.600 \\
(3 \text { Wälle })\end{array}$ & c. 15.000 & 17.058 & Liki $=$ Mackinder-V. \\
\hline 14.250 & - & - & 15.467 & Liki-N \\
\hline$?$ & - & - & c. 15.000 & Sirimon \\
\hline$?$ & - & - & c. 14.500 & Kazita-W \\
\hline 14.200 & - & - & c. 14.600 & Kazita-E \\
\hline 14.300 & $?$ & - & c. 15.300 & $\begin{array}{l}\text { Nithi-N }=\text { Hinde-V. } \\
\quad+\text { Lake Ellis }\end{array}$ \\
\hline- & 一 & - & c. 14.000 & namenloses Zwischental \\
\hline 14.900 (Simba Tarn) & 15.550 (Harris Tarn) & c. $15.700 ?$ & 16.355 & Nithi $=$ Gorges-V. \\
\hline- & - & - & 15.250 & Ruguti-N \\
\hline$?$ & - & - & $"$ & Ruguti \\
\hline- & - & - & ” & Thuchi \\
\hline $\begin{array}{c}14.600(2 \mathrm{x}, 1 \mathrm{x}= \\
\text { Gallery Tarn })\end{array}$ & 15.400 & - & 16.355 & Rupingazi = Hobley-V. \\
\hline- & - & - & c. 15.100 & Nyamindi-E \\
\hline 14.400 (Boden) & - & - & c. 15.300 & Nyamindi \\
\hline$?$ & - & - & c. 15.200 & Nyamindi-W \\
\hline- & - & - & c. 14.100 & Thego \\
\hline $14.200 ?$ & - & - & c. 15.200 & Nairobi = Höhnel-V. \\
\hline $\begin{array}{l}\text { 14.050-14.150 } \\
(2 \text { Wälle })\end{array}$ & $\begin{array}{l}14.200-14.500 \\
\quad(6 \mathrm{Wälle})\end{array}$ & $14.750-15.100$ & 17.058 & $\mathrm{~N}$-Naro-Moru $=$ Teleki-V. \\
\hline 14.200 & 14.350 (2 Wälle) & $14.900-15.050$ & c. 16.050 & Nanyuki = Hausburg-V. \\
\hline ? & - & 一 & c. 14.700 & Nanyuki-N \\
\hline 14.400 & 14.950 & 15.350 & & \\
\hline $\begin{array}{l}550 \\
80 \mathrm{~m}\end{array}$ & $\begin{array}{l}400 \\
60 \mathrm{~m}\end{array}$ & $\begin{array}{l}\text { Summe: } 4.850 \mathrm{ft} . \\
\text { Summe: ü. } 730 \mathrm{~m}\end{array}$ & & \\
\hline
\end{tabular}

Die Höhenangaben Bakens für viele Täler zu IA differieren etwas, Text und Ablesung auf seiner Karte ergeben z. T. verschiedene Höhenwerte; da die Karte 1:25000 nicht so weit reicht, ist eine Entscheidung nicht möglich.

Die von BAKER als IA bezeichneten Stände im Kazita-W und im Ruguti-N dürften zu ID gehören. 
Tabelle 2: Durchschnittliche Gletscheruntergrenzen und $\Delta-$ Werte

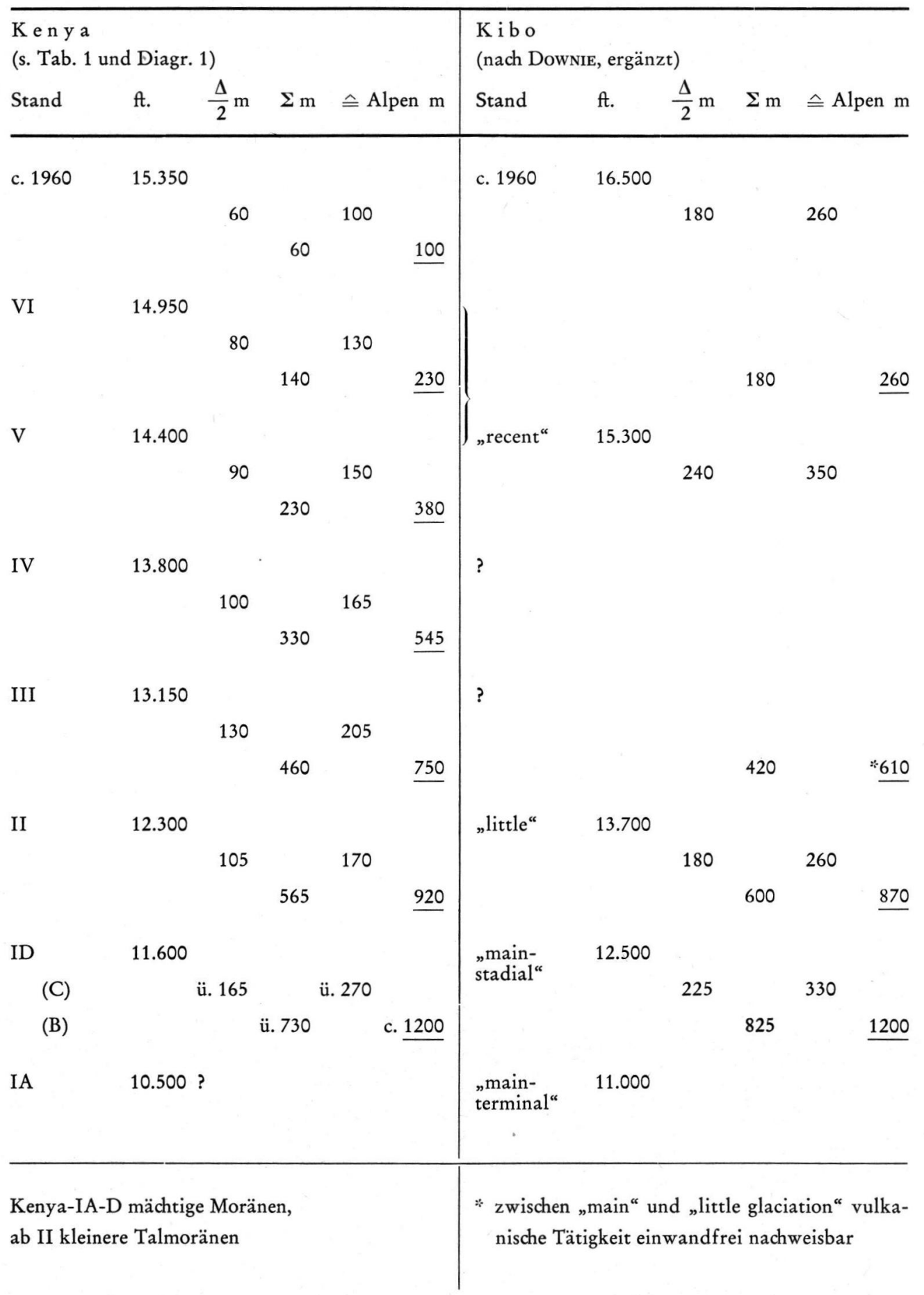


der verschiedenen Rückzugstadien (vergleichbare Werte unterstrichen).

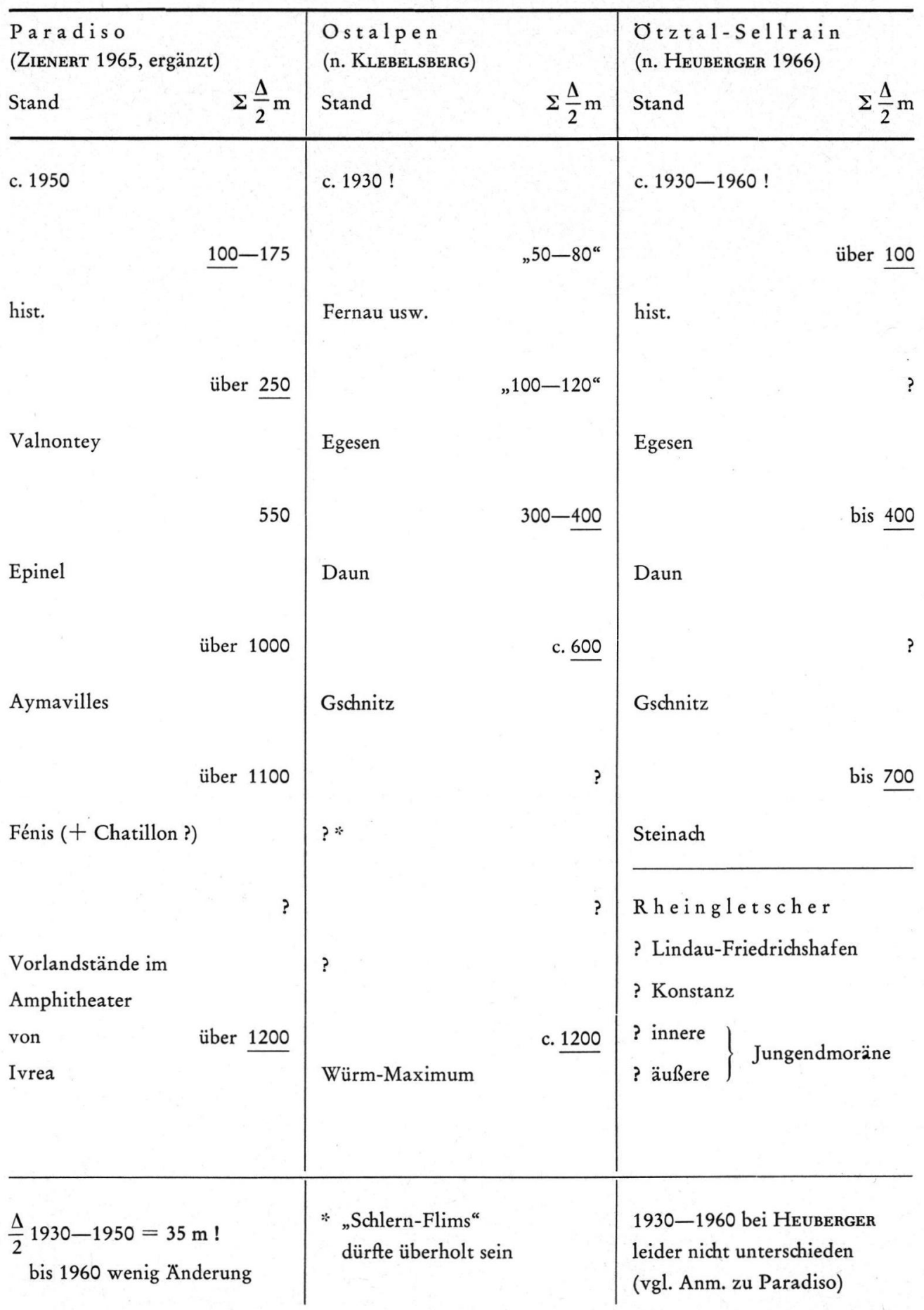




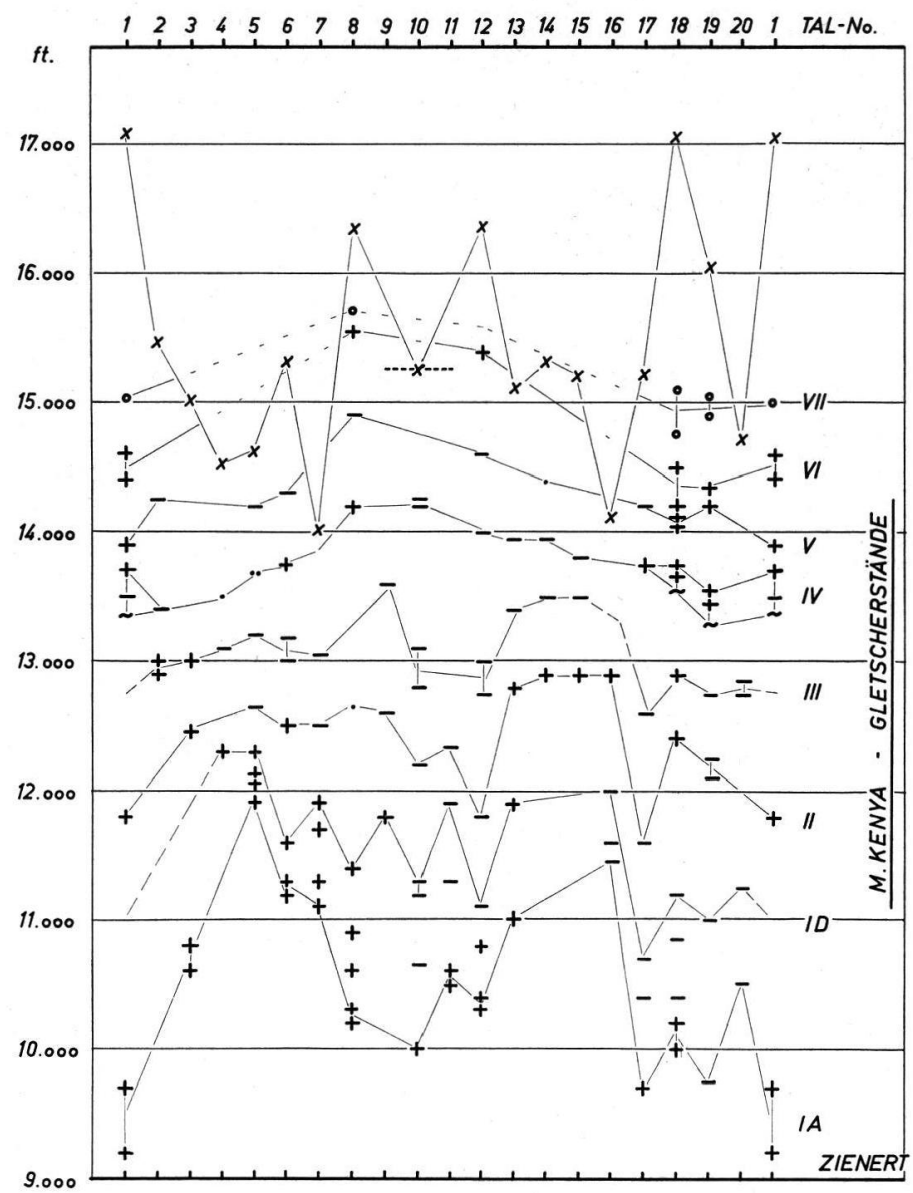

Abb. 1. Gletscherstände der Haupttäler des Mount Kenya (Tab. 1).

+ Moränen nach BAKER 1967; $\approx$ Moränen nach der topogr. Karte 1:25 000, herausgesucht vom Verfasser; ○ Eis c.1960 = „VII“; x höchster Gipfel der Gletscher-Umrandung; • nur Böden erkennbar, keine Wälle; ++ bei IA: verschiedene Angaben bei BAKER, sonst: verschiedene, zumindest höchste und tiefste Werte für einen Stand (soweit darstellbar); Tal-No. siehe Tabelle 1, ebenso die genauen Höhenzahlen.

IC, die bisher nur in einem Teil der Täler nachgewiesen oder wahrscheinlichgemacht werden konnten). Aus diesen Durchschnittswerten kann man die durchschnittlichen $\Delta-$ (= Differenz-) Werte zwischen den Höhen der Moränen der verschiedenen Gletscherstände berechnen. $\Delta / 2$ ergibt dann die jeweilige Veränderung in der Höhenlage der Schneegrenze, ohne daß man auf die leider immer noch meist recht problematische Berechnung der jeweiligen Schneegrenze einzugehen braucht (die Einordnung der einzelnen Moränen in die Gliederung der Gletscherstände ergibt sich ja zwanglos aus dem Diagramm selbst).

Vergleicht man nun die $\Delta / 2$-Werte vom $M$. Kenya mit den aus Mitteleuropa (vor allem den Alpen) bisher bekanntgewordenen Werten für die Schneegrenzdepression der einzelnen Würm-Rückzugsstadien gegenüber heute, so ergeben sich überraschende Parallelen (Tab. 2). Zum besseren Vergleich habe ich dort die Gesamtdepression von über 
$730 \mathrm{~m}$ am Kenya (Diagr. 1) und von $825 \mathrm{~m}$ am Kibo (ähnlich aus einem Diagramm berechnet nach den Angaben bei Ch. DownIE 1964) jeweils auch auf den „alpinen Normalwert" von $1200 \mathrm{~m}$ umgerechnet; entsprechend wurden die übrigen Zahlen behandelt.

Der Vergleich der Verhältnisse am M. Kenya mit denjenigen des Alpengebietes ergibt:

VI entspricht nicht nur nach der Tabelle, sondern auch in der Natur völlig den historischen Moränen unseres Gebietes (Details siehe bei BAKER).

$\mathrm{V}$ dürfte unserem Egesen entsprechen. Der Wert ist etwas niedriger als in meinem Westalpen-Untersuchungsgebiet. Die entsprechenden ostalpinen Werte sind zur Zeit laufenden Änderungen unterworfen, nähern sich aber immer stärker meinen Werten aus dem Paradiso und dem M. Blanc (vgl. z. B. H. Heuberger 1966); die noch bei R. von KLeBelsberg 1948/49 veröffentlichten Werte sind sicher viel zu niedrig.

IV dürfte dem Daun entsprechen. Von hier ab sind aus topographischen Gründen meine Westalpen-Werte nicht mehr vergleichbar, dafür sind für Daun als Durchschnittswerte diejenigen von R. von Klebelsberg gut gesichert und von H. Heuberger 1966 bestätigt.

III käme dann dem Gschnitz gleich und

II dem Steinach im Sinne von H. Heuberger 1966.

Schwierig wird es für die Stände IA-D, hier muß man von unten her versuchen, weiterzukommen. IA ist ja wohl gleich dem Würm-Maximum, dürfte also unserer Äußeren Jungendmoräne entsprechen. Sollte IB der Inneren Jungendmoräne, IC und ID den weiteren Vorland-Rückzugsstadien z. B. des Rheingletschers und des Gletschers der Dora Báltea bei Ivrea entsprechen? Diese Fragen müssen leider z. T. offen bleiben, und zwar einzig und allein deswegen, weil Vieles innerhalb der Außeren Jungendmoränen be i u n s noch nicht genügend geklärt ist, während man es jetzt vom $\mathrm{M}$. Kenya kennt. Eine reine Gebirgs-, ohne Vorland-Vergletscherung bietet hierzu natürlich auch manche Vorteile; leider sind unsere Mittelgebirge für ähnliche Untersuchungen zu niedrig (A. ZIENERT 1967).

Interessante Ergebnisse zeitigt auch der Vergleich mit den von Ch. DownIE veröffentlichten Ergebnissen aus dem Bereich des Kilimandscharo (leider sind diese nicht so exakt wie diejenigen von B. H. BAKER vom Kenya):

Die Moränen der „main glaciation“ umfassen von „main-terminal“ bis „main-stadial“ etwa den Höhenbereich von Kenya-IA bis ID. Die "little glaciation“ scheint zunächst nicht recht $\mathrm{zu}$ passen. Vorher liegt aber eine eindeutig feststellbare vulkanische Tätigkeit des Kibo. Man braucht sich also nur vorzustellen, daß diese Aktivität das Eis weitgehend wegschmolz, so daß die Gletscherkappe sich später erst wieder auf den allmählich auskühlenden, neuen Fördermassen frisch bilden mußte, und schon hat man eine einfache Erklärung dafür, daß der etwas geringere Depressionswert gegenüber heute trotzdem Kenya-II entsprechen dürfte. III und IV scheinen am Kibo zu fehlen, ob aus vulkanischen Gründen, oder nur scheinbar mangels entsprechender Beobachtungen, bleibt unklar. Die „recent glaciation“ am Kibo entspricht zumindest Kenya-V, wahrscheinlich außerdem auch noch VI. Ch. Downie erwähnt zwei Hauptphasen und bis vier Einzelmoränen; nähere Angaben fehlen leider weitgehend.

Faßt man zusammen, so paßt Vieles fast $\mathrm{zu}$ gut zusammen. Es dürfte aber zumindest doch die Mögli ch ke it bestehen, daß hier bei uns und in Ostafrika der Rückgang der Würm-Gletscher durch völlig oder zumindest weitgehend $\mathrm{p}$ a ra 11 el is i e rbare Rückzugsstadien unterbrochen wurde. Und ein solcher Gleichklang der Entwicklung mitten in den Tropen und bei uns wäre doch wirklich ein überraschendes Ergebnis, das eine Nachprüfung auch in anderen Tropengebirgen lohnen dürfte. 


\section{Schrift $\mathrm{cm}$}

BAKER, B. H.: Geology of the Mount Kenya Area. - Geological Survey of Kenya, Report 79, 1967.

DownIE, Ch.: Glaciations of Mount Kilimanjaro, northeast Tanganyika. - Bull. geol. Soc. America 75, 1-16, 1964.

Heuberger, H.: Gletschergeschichtliche Untersuchungen in den Zentralalpen zwischen Sellrainund Ötztal. - Wissensch. Alpenvereinshefte 20, 1966.

KLeBeLsBerg, R. v.: Handbuch der Gletscherkunde und Glazialgeologie. 2 Bde. 1948/49.

Woldstedt, P.: Eiszeitalter. 3 Bde. 1954-65.

Zienert, A.: Gran Paradiso - Mont Blanc: Prähistorische und historische Gletscherstände. - Eiszeitalt. u. Gegenw. 16, 202-225, 1965. - - Vogesen- und Schwarzwald-Kare. - Eiszeitalt. u. Gegenw. 18, 51-75, 1967.

Mount Kenya $1: 25$ 000, DOS 302 (Sonderblatt), 3. Aufl. 1965.

Mount Kenya 1 : 5 000, nur die Gipfelregion, DAV und ƠAV, aufgenommen 1963, o. J.

Mauskript eingeg. 26. 1. 1968.

Anschrift des Verf.: Dr. A. Zienert, 69 Heidelberg 1, Theodor-Körner-Straße 3, ab November 1968: Gerbodoweg 7. 